\title{
MEJORA DE LA ESTRATEGIA DE MUESTREO EN BUCLES DE CONTROL PID BASADOS EN EVENTOS
}

\author{
Oscar Miguel-Escrig (omiguel@uji.es) \\ Julio-Ariel Romero-Pérez (romeroj@uji.es) \\ Departmento de Ingeniería de Sistemas y Diseño. Universitat Jaume I \\ Campus del Riu Sec. Av. Vicent Sos Baynat s/n. \\ CP 12071 Castelló de la Plana. España
}

\section{Resumen}

En este artículo se presenta un estudio sobre el efecto en la robustez y en la generación de eventos de los diferentes métodos de muestreo empleados en el control basado en eventos, a saber, el muestreo por cuantificación regular y el muestreo por cruce de niveles simétricos. Además, se propone como alternativa un nuevo método de muestreo a medio camino entre los anteriores, el cual ofrece un buen compromiso entre robustez y sobrecarga en la generación de eventos.

Palabras clave: PID, control basado en eventos, función descriptiva

\section{INTRODUCCIÓN}

En los últimos años se han realizado numerosos trabajos con diferentes esquemas de muestreo con el fin de aplicarlos al control basado en eventos (CBE) en sistemas continuos. El CBE tiene como virtudes economizar el tráfico de datos que circula por la red y reducir a su vez el uso del actuador del sistema al solo enviar nuevos datos al controlador cuando se produce un cambio significativo en el sistema, en contraposición a los controladores clásicos donde la acción de control es calculada periódicamente, independientemente de las necesidades del sistema.

Dentro del CBE, las diferentes estrategias de muestreo cobran un protagonismo especial ya que en muchos casos son estas propias estrategias las encargadas de generar los eventos para la ejecución del controlador. Entre ellas las que más destacan son aquellas en las que la generación de eventos es debida al cruce de ciertos niveles o umbrales.

Una de las estrategias de este tipo más trabajadas en los últimos tiempos es la estrategia basada en enviar datos siempre y cuando la señal de error supere un valor $\delta$, conocida por las siglas SOD (del inglés Send-On-Delta), cuya eficacia en reducir el tráfico de datos ha sido ampliamente probada $[3,4]$, y más concretamente una variante de esta caracterizada por incluir una histéresis con el mismo valor $\delta$ conocida como SSOD (del inglés
Symmetric-Send-On-Delta), presentado en [2].

Con respecto a esta estrategia de muestreo, numerosos trabajos han sido presentados en la última década, entre los que cabe destacar las aportaciones mostradas en [5] donde se propone una regla simple para la sintonización de controladores continuos usados en este esquema de muestreo con el fin de dotarlos de cierta robustez.

Otra posible estrategia de muestreo es el cuantificador regular (RQ, del inglés Regular Quantifier), donde el envío de datos se produce cuando la señal de error es múltiplo de cierto valor, siendo así muy fácilmente implementables.

En [6] se recoge un estudio comparando estas dos últimas estrategias de muestreo comentadas, donde los autores detallan algunas de las principales características de los sistemas controlados mediante estos métodos de muestreo tanto en términos de robustez como de desempeño del sistema.

En el presente trabajo se propone un nuevo método de generación de eventos que incluye como casos particulares el SSOD y el RQ. En este sentido el método propuesto es una generalización que ofrece un compromiso entre las principales virtudes de cada uno de ellos.

El artículo se estructura como sigue: en la sección 2 se hace un breve resumen sobre las principales características de los métodos de muestreo y se introduce la alternativa planteada. En la sección 3 se ofrece una caracterización de la robustez de los diferentes métodos de muestreo empleados por medio del uso de la función descriptiva. Seguidamente, en la sección 4 se presentan algunos ejemplos mediante simulación con las diferentes estrategias de muestreo planteadas. Finalmente, en la sección 5 se exponen las conclusiones sobre este trabajo.

\section{PROPUESTA DE MUESTREO}

Considere el sistema de control en red mostrado en la Figura 1 donde $C(s)$ y $G(s)$ son las funciones de transferencia del controlador y del proceso respectivamente, el bloque EG representa el generador de eventos y el bloque $\mathrm{ZOH}$ un retenedor de or- 


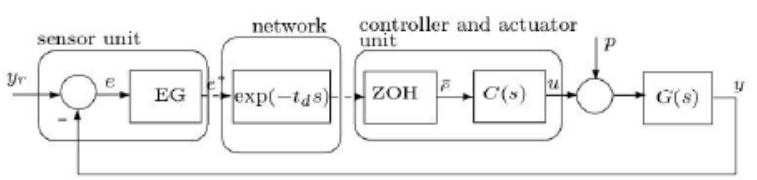

Figura 1: Esquema de bucle de control PID basado en eventos propuesto en [2].

den cero. Además $y_{r}$ es la referencia, $y$ es la salida controlada y $p$ es una entrada de perturbación.

Este esquema de control fue propuesto en [2] considerando que $C(s)$ es un controlador PI y que el generador de eventos se basa en un muestreo tipo SSOD, por ello, los autores la llamaron arquitectura SSOD-PI. Este trabajo no se centra en el estudio de los controladores PI ni se limita al mecanismo SSOD, por lo que podemos nombrar en general las arquitecturas que se van a tratar con el nombre $E G-C(s)$.

En el esquema presentado en la Figura 1 se asume que el controlador está localizado cerca del actuador y que el sensor envía medidas $e^{*}$ del error $e=y_{r}-y$ al controlador a través de una red de comunicaciones. El envío se realiza mediante el bloque generador de eventos (EG). El bloque ZOH mantiene en $\bar{e}$ el último valor de $e^{*}$ hasta que un nuevo valor sea enviado.

Como se ha comentado anteriormente los dos principales métodos de muestreo usados para la generación de eventos por cruce de niveles son el muestreador SSOD y el RQ.

El muestreador SSOD se caracteriza por enviar un nuevo dato cada vez que la señal muestreada cambia en un valor $\delta$ con respecto a la última muestra enviada, como se muestra en la Figura 2.

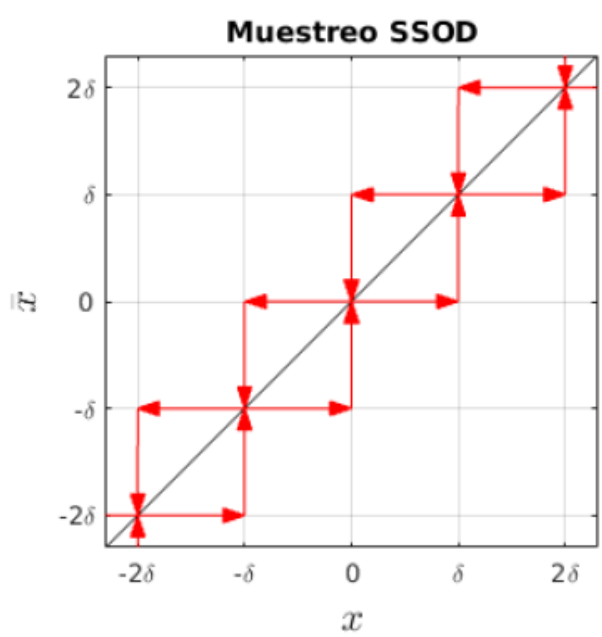

Figura 2: Muestreo SSOD.

Este tipo de muestreo presenta un buen rechazo a la generación de eventos debidos al ruido en la señal muestreada, ya que la histéresis que se incluye evita la aparición de dichos eventos totalmente siempre y cuando el ruido medido no tenga una amplitud mayor a $\delta$.

Sin embargo, y debido también a la misma histéresis, las arquitecturas que contienen al SSOD pueden propiciar la aparición de ciclos límite, necesitando generalmente controladores bastante robustos. Una regla bastante simple en cuanto a su sintonización se introduce en [5], donde se recomiendan márgenes de fase mayores a $45^{\circ}$.

En cuanto al muestreador RQ, este se caracteriza por enviar un nuevo dato cada vez que la señal a muestrear cruza un nivel sin tener memoria de los anteriores cruces producidos, tal y como se muestra en la Figura 3.

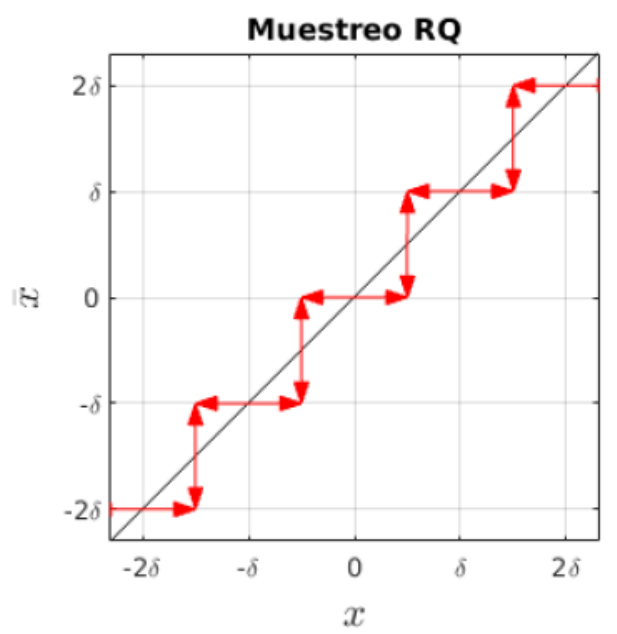

Figura 3: Muestreo RQ.

Esta estrategia permite el uso de controladores más rápidos que los usados en las arquitecturas con SSOD debido a la falta de histéresis que dificulte la reacción del controlador, pero, sin embargo, presenta la desventaja de ser muy sensible al ruido, produciendo una tasa de generación de eventos extremadamente elevada si la señal muestreada es muy ruidosa.

Teniendo en cuenta los puntos fuertes y débiles de cada una de las estrategias expuestas, se propone usar una estrategia de muestreo intermedia, donde la generación de eventos será como la del muestreo SSOD pero no siendo simétrica la histéresis, como se muestra en la Figura 4. Cabe destacar, que el muestreo RQ es equivalente al muestreo SSOD pero con valor de histéresis 0 , de ahí que el método propuesto lo consideremos un caso intermedio.

Este nuevo esquema de muestreo intermedio entre el SSOD y el RQ presenta virtudes y defectos de ambos a medida que sea más próximo a uno u otro. $\mathrm{Al}$ incluir una histéresis al muestreador RQ se está 


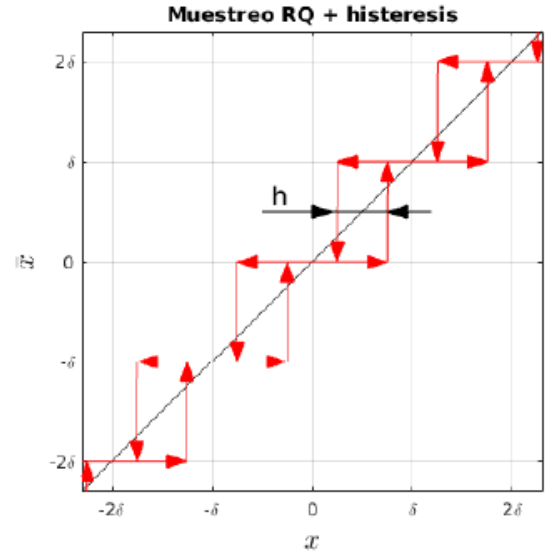

Figura 4: Muestreo RQ con histéresis.

evitando la generación de eventos debidos al ruido pero a su vez se está perjudicando la rapidez que el controlador puede llegar a tener. Y a la inversa, reducir la histéresis del SSOD contribuye a reducir el desfase en la señal que este muestreo introduce, aumenta la velocidad que los controladores pueden llegar a tener debido a la reducción de robustez necesaria y todo a cambio de reducir el ancho del ruido que puede llegar a rechazar.

Para ilustrar el efecto de las diferentes técnicas de muestreo comentadas se incluye un ejemplo en la Figura 5, donde el muestreador SSOD, RQ y dos muestreadores con diferente valor de histéresis muestrean una señal senoidal a la que se añade ruido. Los muestreadores tienen un ratio entre el ancho de histéresis $(h)$ y $\delta$ de $1 / 3$ y $2 / 3$ respectivamente. En esa misma figura, se puede apreciar que el número de eventos generado por los diferentes muestreadores aumenta a medida que se reduce el valor de la histéresis y que al aumentar el valor de la histéresis se incrementa también el retraso de la señal muestreada.

\section{EFECTO SOBRE LA ROBUSTEZ FRENTE A CICLOS LÍMITES}

Tal y como se ha comentado con anterioridad, estos esquemas de muestreo tienen una influencia muy importante sobre la robustez respecto a la aparición de ciclos límite de los sistemas en los que se aplican. Esta robustez se puede caracterizar mediante el uso de técnicas de análisis como la función descriptiva.

Para aplicar el método de la función descriptiva en este tipo de sistemas solo hay que tener en cuenta que todos los bloques que se encuentran antes del sistema en la Figura 1 pueden agruparse en un solo bloque tal y como se muestra en la Figura 6
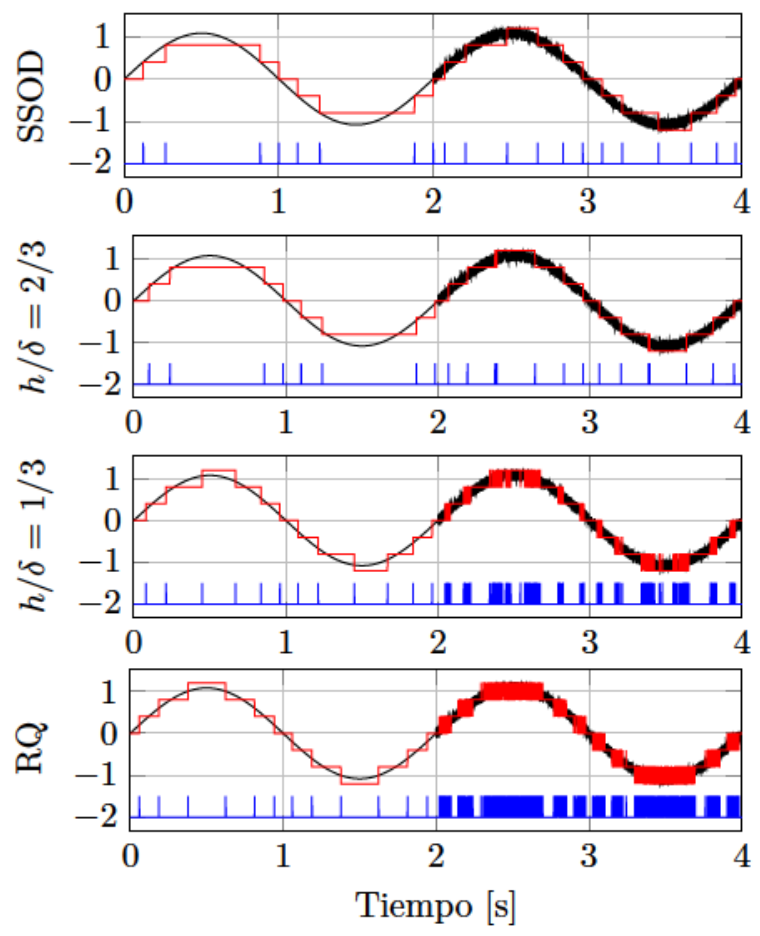

Figura 5: Muestreo (rojo) de una señal seno, con y $\sin$ ruido (negro) con los respectivos eventos (azul).

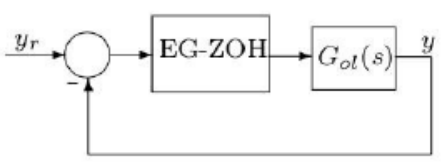

Figura 6: Diagrama de bloques equivalente al esquema de control de la Figura 1.

con el bloque $E G-Z O H$, el cual es tratado como la no-linealidad a estudiar.

Es sabido que la condición para la existencia de ciclos límite en el esquema de la Figura 6 viene dada por la ecuación (1), donde $G_{o l}$ representa la función de transferencia en bucle abierto y $N$ es la función descriptiva de la no-linealidad. Por tanto, el sistema no presentará ciclos límite si los gráficos de $G_{o l}(j \omega)$ y $-\frac{1}{N}$ en el diagrama de Nyquist no se intersectan.

$$
G_{o l}(j \omega)=-\frac{1}{N} ; \quad \forall \omega
$$

Con el fin de estudiar la robustez de un muestreador en función del cociente $h / \delta$, se han obtenido gráficas de $-\frac{1}{N}$ para valores de $h / \delta$ comprendidos entre 0 (RQ) y 1 (SSOD). En la Figura 7 se representa la evolución en función $h / \delta$ de una parte la función descriptiva, en concreto la relacionada con las oscilaciones que provocan la conmutación de $e^{*}$ entre dos niveles. Como se puede ver, la modifica- 


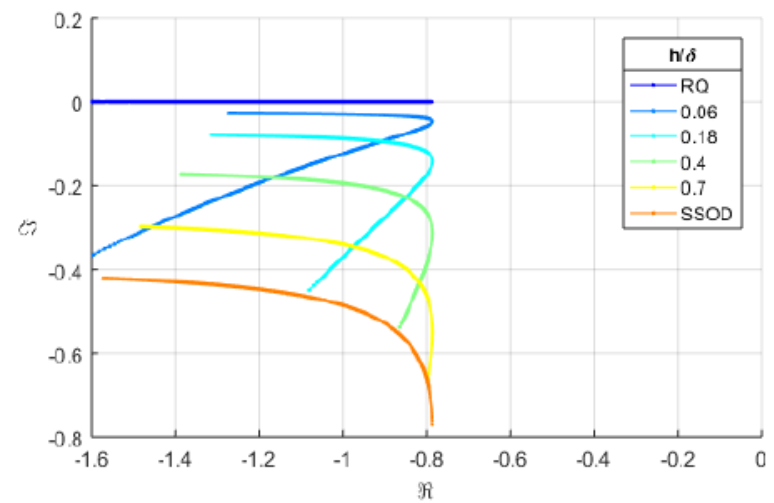

Figura 7: Evolución de la función descriptiva para oscilaciones de un solo nivel entre un muestreador RQ y un SSOD.

ción de la relación entre el valor de $\delta$ y $h$ afecta de forma considerable la forma de la $-\frac{1}{N}$, siendo una recta sobre el eje real negativo para $h=0 \mathrm{y}$ curvas en el tercer cuadrante cuya forma depende de $h / \delta$ para $h \neq 0$.

En la Figura 8 se puede observar la función descriptiva completa para muestreadores con cuatro valores diferentes de $h / \delta: 0,1 / 3,2 / 3$ y 1 . También se muestra la forma de una hipotética función de transferencia de bucle abierto $G_{o l}$.
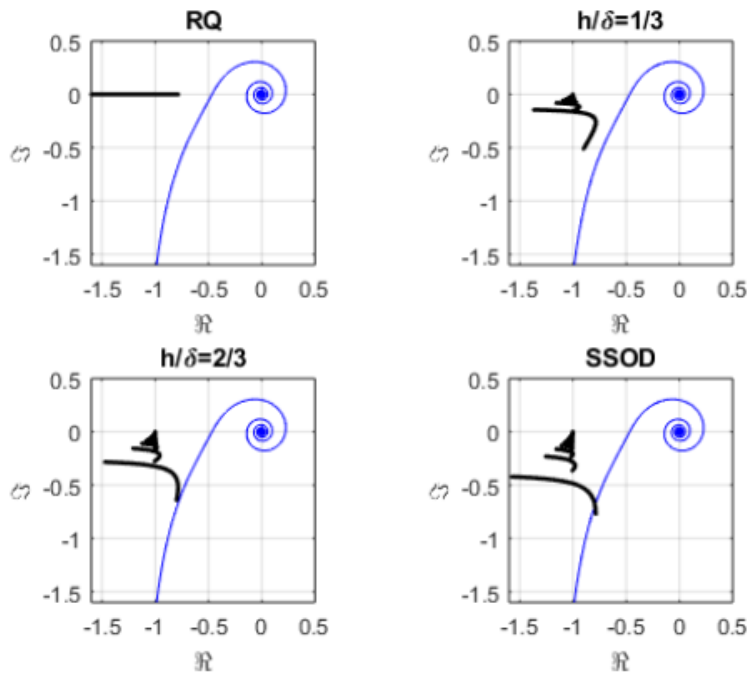

Figura 8: Funciones descriptivas completas de los diferentes muestreadores (negro). Función de transferencia de bucle abierto $G_{o l}$ hipotética (azul).

Gráficamente se puede interpretar que para el caso mostrado en la Figura 8 los lazos que contienen al muestreador RQ y a los dos casos intermedios no oscilarán pero sí lo hará el que contiene el SSOD. Sin embargo, entre los dos casos intermedios, el caso con $h / \delta=2 / 3$ estará mucho más cerca de oscilar porque su función descriptiva está muy próxi-
Tabla 1: Resumen de los parámetros de los controladores empleados.

\begin{tabular}{|c|c|c|c|c|}
\cline { 2 - 5 } \multicolumn{1}{c|}{} & $K_{p}$ & $T_{i}$ & $T_{d}$ & $N$ \\
\hline$G_{1}$ & 0.885 & 0.953 & 0.443 & 10 \\
\hline$G_{2}$ & 0.983 & 1.053 & 0.471 & 10 \\
\hline
\end{tabular}

ma a la función de transferencia en bucle abierto.

\section{SIMULACIONES}

Para poner en evidencia lo anteriormente comentado sobre los diferentes esquemas de muestreo mostrados se proponen dos sistemas de segundo orden con retardo, mostrados en (2), sobre los que se va a realizar el control en bucle cerrado con la ayuda de un controlador PID.

$$
G_{1}(s)=\frac{e^{-s}}{(1+0,6 s)^{2}} ; \quad G_{2}(s)=\frac{e^{-s}}{(1+0,7 s)^{2}}
$$

La sintonización de los controladores se ha realizado usando el método AMIGO presentado en [1]. Para el diseño se ha usado como valor máximo de la función de sensibilidad $M_{s}=2 \mathrm{y}$ un coeficiente de filtro del término derivativo $N=10$. Los valores obtenidos se recogen en la Tabla 1.

Aplicando los criterios de estabilidad de la función descriptiva sobre el conjunto de muestreadores y con el sistema $G_{1}$ podemos observar en la Figura 9 que solamente la arquitectura que contiene el muestreador SSOD intersecta con la función descriptiva de la no-linealidad, por lo tanto, esta será la única arquitectura para la cual existirán oscilaciones.

Para comprobar estos resultados se ha realizado una simulación donde el sistema se somete a un cambio de referencia, una vez estabilizado se incorpora una perturbación de tipo escalón y finalmente se simula el efecto de tener un error de medida sobre el sistema. Los resultados de dicha simulación se pueden observar en la Figura 10, donde efectivamente comprobamos que solamente el sistema con el SSOD presenta ciclos límite.

De igual modo, para el sistema $G_{2}$ se ha procedido a evaluar su comportamiento en el plano de Nyquist. Para este sistema se puede observar en la Figura 11 que su función de transferencia en bucle abierto intersecta a la función descriptiva del muestreador SSOD y del muestreador con $h / \delta=2 / 3$, por lo tanto, es de esperar que para estas dos arquitecturas el sistema oscile.

Realizando una simulación con las mismas condiciones que para el caso del primer sistema, se pue- 

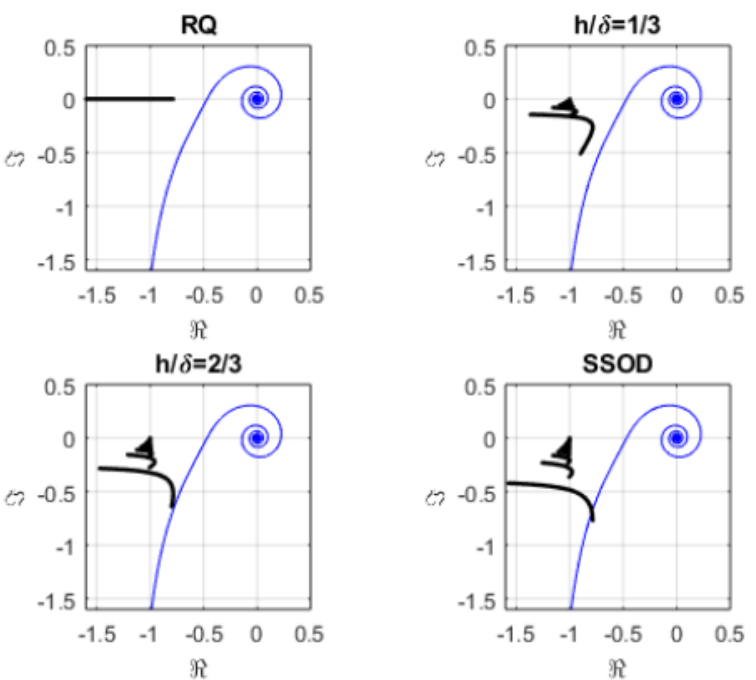

Figura 9: Representación de las funciones descriptivas de los muestreadores y de la función de bucle abierto de $G_{1}$.

de observar en la Figura 12 que tanto el lazo que contiene el SSOD como el lazo con el muestreador con $h / \delta=2 / 3$ presentan un ciclo límite.

Además, a medida que la histéresis se reduce se puede observar cualitativamente, en los casos donde no hay oscilaciones, que la generación de eventos debida al ruido es mayor.

Finalmente, sobre la estabilidad de los sistemas, cabe destacar que la cercanía de la función de transferencia a la función descriptiva puede inducir a que en casos donde están muy cerca de intersectarse, como el presentado para el primer sistema con el muestreador con $h / \delta=2 / 3$ (ver Figura 9), la propia adición de ruido propicie la aparición de algunas oscilaciones no deseadas como se muestra en la Figura 13.

\section{CONCLUSIONES}

En este artículo se ha estudiado el efecto de aplicar diferentes esquemas de muestreo sobre la robustez de un sistema, partiendo de los esquemas de muestreo más conocidos, como lo son el muestreador SSOD o el muestreador RQ. Se han detallado las virtudes e inconvenientes de estos dos esquemas de muestreo y se ha propuesto una graduación entre ellos.

Con el fin de evaluar la nueva propuesta de muestreo se han utilizado técnicas de análisis de sistemas como la función descriptiva, la cual ha sido obtenida y representada tanto para los casos extremos, a saber, RQ y SSOD, como para los casos intermedios.

Finalmente, se han realizado diferentes experien-
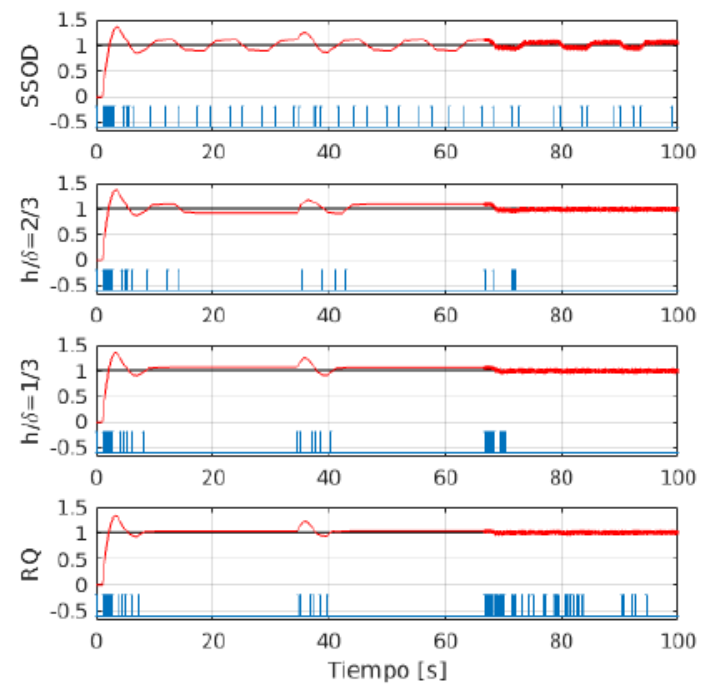

Figura 10: Respuesta temporal (rojo) y eventos generados (azul) en $G_{1}$ frente a cambio de referencia (negro), perturbación y ruido.

cias con algunos sistemas poniendo de manifiesto las características principales métodos de muestreo y mostrando la conveniencia de obtener métodos de muestreo intermedios para aprovechar al máximo las virtudes de cada uno.

\section{Agradecimientos}

Este trabajo ha sido financiado mediante los proyectos TEC2015-69155-R del MINECO y P1·1B2015-42 de la Universitat Jaume I.

\section{English summary}

IMPROVEMENT OF THE SAMPLING STRATEGY IN EVENTBASED PID CONTROL LOOPS

\section{Abstract}

In this paper a study of the effect on the robustness and the event generation of different sampling methods usually used in event-based control is presented. These methods, namely the regular quantifier and the symmetric-send-on-delta, have some disadvantages that are tried to be resolved by the new sampling method proposed in this paper, which offers a good balance between robustness and event bursts.

Keywords: SSOD, RQ, Describing Function, EBC 

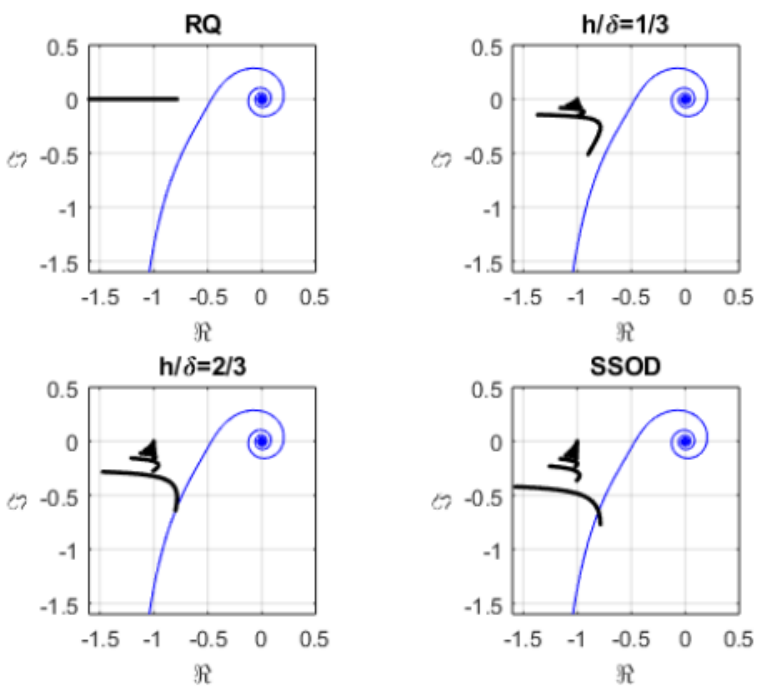

Figura 11: Representación de las funciones descriptivas de los muestreadores y de la función de bucle abierto de $G_{2}$.

\section{Referencias}

[1] K.J. Åström and T. Hägglund. Revisiting the Ziegler-Nichols step response method for PID control. Journal of Process Control, 14(6):635-650, sep 2004.

[2] M. Beschi, S. Dormido, J. Sanchez, and A. Visioli. Characterization of symmetric send-ondelta pi controllers. Journal of Process Control, 22(10):1930-1945, 2012.

[3] S. Dormido, J. Sánchez, and E. Kofman. Muestreo, control y comunicación basados en eventos. Revista Iberoamericana de Automática e Informática Industrial RIAI, 5(1):5-26, 2008.

[4] J. Ploennigs, V. Vasyutynskyy, and K. Kabitzsch. Comparative study of energy-efficient sampling approaches for wireless control networks. IEEE Transactions on Industrial Informatics, 6(3):416-424, 2010.

[5] J. A. Romero Pérez and R. Sanchis Llopis. A new method for tuning pi controllers with symmetric send-on-delta sampling strategy. ISA transactions, 64:161-173, 2016.

[6] J. A. Romero Pérez and R. Sanchis Llopis. Tuning and robustness analysis of eventbased pid controllers under different eventgeneration strategies. International Journal of Control, pages 1-21, 2017.
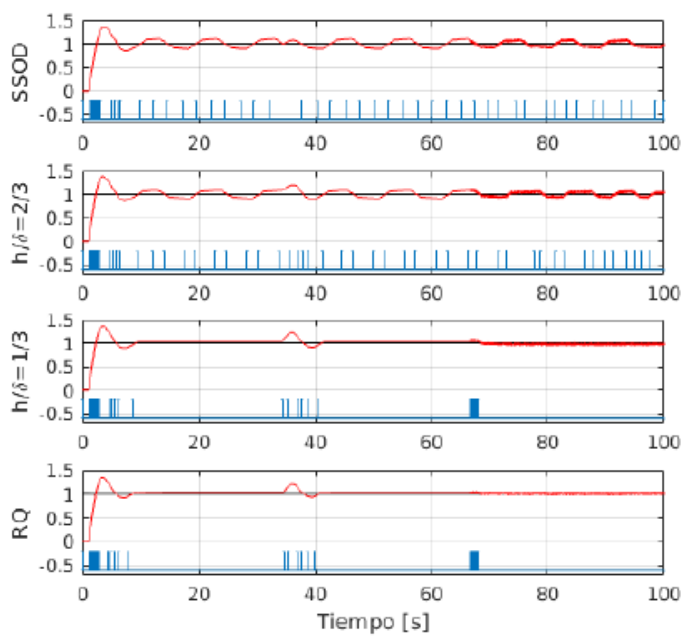

Figura 12: Respuesta temporal (rojo) y eventos generados (azul) en $G_{2}$ frente a cambio de referencia (negro), perturbación y ruido.
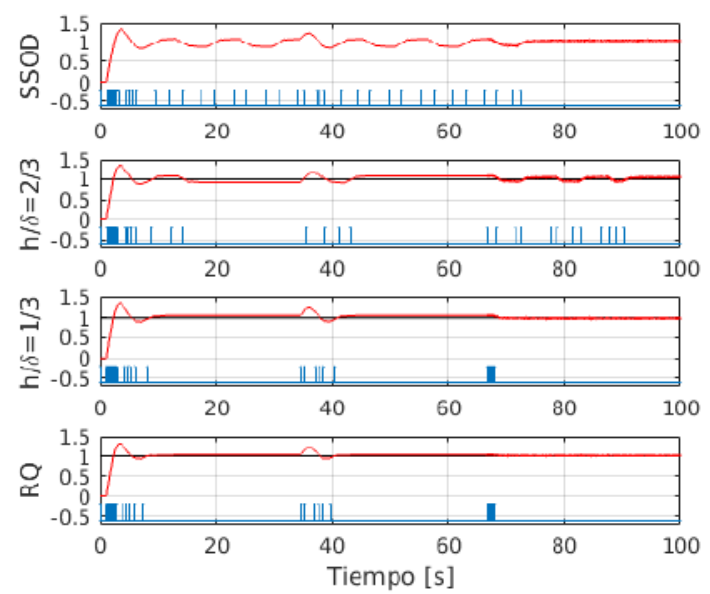

Figura 13: Caso especial de la respuesta temporal (rojo) y eventos generados (azul) en $G_{1}$ frente a cambio de referencia (negro), perturbación y ruido.

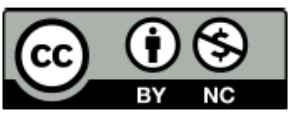

(C) 2018 by the authors. Submitted for possible open access publication under the terms and conditions of the Creative Commons Attribution CC-BY-NC 3.0 license (http://creativecommons.org/licenses/by-nc/3.0/). 